\title{
Type 1 diabetes defined by severe insulin deficiency occurs after 30 years of age and is commonly treated as type 2 diabetes
}

\author{
Nicholas J. Thomas ${ }^{1,2} \cdot$ Anita L. Lynam ${ }^{1} \cdot$ Anita V. Hill ${ }^{1} \cdot$ Michael N. Weedon ${ }^{1} \cdot$ Beverley M. Shields ${ }^{1}$ • \\ Richard A. Oram ${ }^{1,3} \cdot$ Timothy J. McDonald ${ }^{1,4}$. Andrew T. Hattersley ${ }^{1,2}$. Angus G. Jones ${ }^{1,2}$
}

Received: 8 January 2019 / Accepted: 22 February 2019 / Published online: 10 April 2019

(C) The Author(s) 2019

\begin{abstract}
Aims/hypothesis Late-onset type 1 diabetes can be difficult to identify. Measurement of endogenous insulin secretion using Cpeptide provides a gold standard classification of diabetes type in longstanding diabetes that closely relates to treatment requirements. We aimed to determine the prevalence and characteristics of type 1 diabetes defined by severe endogenous insulin deficiency after age 30 and assess whether these individuals are identified and managed as having type 1 diabetes in clinical practice.

Methods We assessed the characteristics of type 1 diabetes defined by rapid insulin requirement (within 3 years of diagnosis) and severe endogenous insulin deficiency (non-fasting C-peptide $<200$ pmol/l) in 583 participants with insulin-treated diabetes, diagnosed after age 30, from the Diabetes Alliance for Research in England (DARE) population cohort. We compared characteristics with participants with retained endogenous insulin secretion $(>600 \mathrm{pmol} / \mathrm{l})$ and 220 participants with severe insulin deficiency who were diagnosed under age 30.

Results Twenty-one per cent of participants with insulin-treated diabetes who were diagnosed after age 30 met the study criteria for type 1 diabetes. Of these participants, 38\% did not receive insulin at diagnosis, of whom 47\% self-reported type 2 diabetes. Rapid insulin requirement was highly predictive of severe endogenous insulin deficiency: 85\% required insulin within 1 year of diagnosis, and $47 \%$ of all those initially treated without insulin who progressed to insulin treatment within 3 years of diagnosis had severe endogenous insulin deficiency. Participants with late-onset type 1 diabetes defined by development of severe insulin deficiency had similar clinical characteristics to those with young-onset type 1 diabetes. However, those with later onset type 1 diabetes had a modestly lower type 1 diabetes genetic risk score $(0.268$ vs $0.279 ; p<0.001$ [expected type 2 diabetes population median, 0.231]), a higher islet autoantibody prevalence (GAD-, islet antigen 2 [IA2]- or zinc transporter protein 8 [ZnT8]positive) of $78 \%$ at 13 years vs $62 \%$ at 26 years of diabetes duration; $(p=0.02)$, and were less likely to identify as having type 1 diabetes $(79 \%$ vs $100 \% ; p<0.001)$ vs those with young-onset disease.

Conclusions/interpretation Type 1 diabetes diagnosed over 30 years of age, defined by severe insulin deficiency, has similar clinical and biological characteristics to that occurring at younger ages, but is frequently not identified. Clinicians should be aware that patients progressing to insulin within 3 years of diagnosis have a high likelihood of type 1 diabetes, regardless of initial diagnosis.
\end{abstract}

Keywords Autoantibodies $\cdot$ Classification $\cdot$ C-peptide $\cdot$ Genetic risk score $\cdot$ Type 1 diabetes $\cdot$ Type 2 diabetes

Electronic supplementary material The online version of this article (https://doi.org/10.1007/s00125-019-4863-8) contains peer-reviewed but unedited supplementary material, which is available to authorised users.

Angus G. Jones

angus.jones@exeter.ac.uk

1 Institute of Biomedical and Clinical Science, University of Exeter Medical School, Barrack Road, Exeter EX25DW, UK

2 Department of Diabetes and Endocrinology, Royal Devon and Exeter NHS Foundation Trust, Exeter, UK
3 Renal Department, Royal Devon and Exeter NHS Foundation Trust, Exeter, UK

4 Academic Department of Blood Sciences, Royal Devon and Exeter NHS Foundation Trust, Exeter, UK 


\section{Research in context}

\section{What is already known about this subject?}

- Differentiating type 1 from type 2 diabetes when diagnosed over 30 years of age is difficult

- Recent research using novel genomic methods has suggested that over $42 \%$ of type 1 diabetes occurs after age 30 , and the phenotype remains severe, with $89 \%$ being insulin treated within 1 year of diagnosis

- Severe insulin deficiency is a hallmark of type 1 diabetes and defines treatment and education requirements

\section{What is the key question?}

- What is the prevalence and characteristics of type 1 diabetes defined by severe endogenous insulin deficiency diagnosed after age 30 ?

\section{What are the new findings?}

- Twenty-one per cent of participants with insulin-treated diabetes, diagnosed after age 30 , had severe insulin deficiency. These participants had similar clinical, islet autoantibody and genetic characteristics to a comparison cohort with young-onset type 1 diabetes

- Thirty-eight per cent of those with severe insulin deficiency did not receive insulin treatment at diagnosis

- Rapid insulin requirement was highly predictive of severe endogenous insulin deficiency: $85 \%$ required insulin within 1 year of diagnosis

\section{How might this impact on clinical practice in the foreseeable future?}

- This study provides clear evidence that type 1 diabetes leading to severe, near absolute, insulin deficiency is common in later life but is often unrecognised. Clinicians should suspect type 1 diabetes where a patient requires insulin treatment within 3 years of diagnosis

\section{Abbreviations \\ DARE Diabetes Alliance for Research in England \\ T1DGRS Type 1 diabetes genetic risk score}

\section{Introduction}

Type 1 diabetes is classically defined by autoimmune or idiopathic beta cell destruction leading to severe insulin deficiency [1], but this aetiopathological definition is difficult to apply in clinical practice. Definitions based on clinical criteria have been little investigated in adults and are poorly defined, with many features commonly thought of as discriminatory, having no evidence base [2]. As a result, there is no robust evidencebased guidance on identifying type 1 diabetes in later life. Recent novel population-based genetic stratification analysis has suggested that at least $42 \%$ of type 1 diabetes occurs after the age of 30 [3]. This research suggests late type 1 diabetes has similar characteristics to young-onset disease and, in contrast to type 1 diabetes defined by autoantibody status alone, continues to have a severe phenotype: $89 \%$ were treated with insulin within 1 year of diagnosis, and $11 \%$ developed ketoacidosis [4]. However, this technique does not allow classification of type 1 diabetes at an individual level, and neither islet autoantibodies nor measures of endogenous insulin secretion were available in the UK Biobank population analysed [3].

Measurement of C-peptide, a surrogate marker of insulin secretion, allows robust diagnosis of type 1 diabetes in longstanding diabetes ( $>3$ years duration) and closely relates to treatment requirements [5]. The development of severe (near absolute) insulin deficiency (commonly defined by stimulated C-peptide $<200 \mathrm{pmol} / \mathrm{l}$ ) results in high glucose variability, marked hypoglycaemia risk, absolute insulin requirement and poor glycaemic response to non-insulin therapies $[5,6]$. Therefore, those with severe insulin deficiency will require glycaemic management according to type 1 diabetes guidelines regardless of disease aetiology. The prevalence and characteristics of diabetes leading to severe insulin deficiency in older adults is not known.

We aimed to determine the prevalence and characteristics of type 1 diabetes defined by severe endogenous insulin deficiency in patients $>30$ years of age with insulin-treated diabetes. We also assessed whether these patients were identified and managed as having type 1 diabetes in clinical practice.

\section{Methods}

We assessed the prevalence and characteristics of type 1 diabetes defined by early insulin requirement and severe 
endogenous insulin deficiency in an insulin-treated population cohort.

Participants 583 participants from the population-based Exeter Diabetes Alliance for Research in England (DARE) cohort met the following inclusion criteria: diagnosed with diabetes after 30 years of age, insulin treated and with a Cpeptide measurement available. Participants with previous pancreatic pathology $(n=2)$ were excluded from analysis. To allow for comparison with young-onset type 1 diabetes, we assessed a further cohort of 220 DARE participants with age of diabetes diagnosis $\leq 30$ years who met the study criteria for type 1 diabetes (ESM Fig. 1; see below).

The DARE study was approved by the South West ethics committee (UK). Participants gave informed consent.

Assessment of clinical characteristics Clinical history was selfreported by participants in an interview with a research nurse, and height and weight were measured at a median diabetes duration of 13 years. Time to insulin was defined as immediate if within 2 weeks of diagnosis.

Laboratory analysis Non-fasting (random) C-peptide, islet autoantibodies (GAD, zinc transporter protein 8 [ZnT8], islet antigen 2 [IA2]) and a type 1 diabetes genetic risk score (T1DGRS) were assessed in all included participants, as previously described (see ESM methods) [6, 7]. Where multiple C-peptide measurements were available (70\% of participants, median 3 values per participant), the median value was used. Median duration at islet autoantibody and C-peptide assessment was 13 and 16 years respectively (ESM Methods).

Definition of diabetes type Type 1 diabetes was defined as continuous insulin treatment commenced within 3 years of diagnosis and severe insulin deficiency defined by a nonfasting C-peptide $<200 \mathrm{pmol} / \mathrm{l}$. Insulin-treated type 2 diabetes was defined as current insulin treatment with a $\mathrm{C}$-peptide $\geq 600 \mathrm{pmol} / 1$ and a duration of diabetes of over 3 years at Cpeptide measurement. Participants who were insulin treated and with a C-peptide level $\geq 200-<600 \mathrm{pmol} / 1$ ( $n=115$, median 48 months from diagnosis to insulin therapy) were considered indeterminate and were not included in analysis (ESM Fig. 1) [5].

Statistical analysis Data were assessed visually for distribution. Data for all continuous variables except age were not normally distributed; therefore, data are presented as median and interquartile range (IQR), unless otherwise stated. We compared the clinical characteristics, islet autoantibody status and T1DGRS of participant groups defined by C-peptide, initial insulin treatment (within 2 weeks of diagnosis) and age at diagnosis using the Wilcoxon rank-sum test for continuous variables and $\chi^{2}$ analysis for comparison of categorical characteristics. All analyses were performed using Stata 15 (StataCorp LP, College Station, TX, USA).

\section{Results}

Severe insulin deficiency occurs in $21 \%$ of insulin-treated patients diagnosed after 30 years of age and has similar clinical characteristics to young-onset type 1 diabetes Twenty-one per cent (123/583) of insulin-treated participants diagnosed with diabetes after 30 years of age met the study criteria for type 1 diabetes and had severe endogenous insulin deficiency (insulin treatment within 3 years and C-peptide $<200 \mathrm{pmol} / \mathrm{l}$ ) (ESM Fig. 1).

The characteristics of participants with late-onset (>30 years of age) type 1 diabetes, young-onset ( $\leq 30$ years of age) type 1 diabetes and late-onset type 2 diabetes (retained endogenous insulin secretion) are shown in Table 1. Participants with late-onset type 1 diabetes defined by development of severe insulin deficiency had broadly similar characteristics to those with young-onset type 1 diabetes: BMI, insulin dose and $\mathrm{HbA}_{1 \mathrm{c}}$ did not differ. However, those with later onset type 1 diabetes had a modestly lower T1DGRS ( 0.268 vs 0.279 [expected type 2 diabetes population median 0.231 [8]]), higher islet autoantibody prevalence (78\% vs $62 \%$, at 13 vs 26 years duration) and were more likely to be treated as, and identify as having, type 2 diabetes (oral glucose-lowering agent use, $15 \%$ vs $5 \%$; insulin at diagnosis, $62 \%$ vs $96 \%$; self-reported type 2 diabetes $20 \%$ vs $0 \%$ ).

Classical clinical criteria cannot reliably identify individuals with late-onset type 1 diabetes leading to severe insulin deficiency Despite similar clinical features to young-onset type 1 diabetes, classical clinical criteria could not robustly identify late-onset type 1 diabetes. Only $41 \%$ had a BMI $<25$ and $28 \%$ of participants with a BMI $<25$ had type 2 diabetes. UK National Institute for Health and Care Excellence guidance for type 1 diabetes identification (age of diagnosis $<50$ or BMI $<25 \mathrm{~kg} / \mathrm{m}^{2}$ ) identified $81 \%$ of type 1 diabetes cases but had very low specificity $(41 \%)$. The specificity of these criteria would be far lower had non-insulin treated individuals been included in this cohort.

Thirty-eight per cent of participants with late-onset type 1 diabetes did not receive insulin at diagnosis Participants meeting criteria for type 1 diabetes after age 30 who did not receive insulin at diagnosis $(38 \%)$ commenced insulin a median of 12 months from diagnosis, and had similar characteristics to those commencing insulin at diagnosis (ESM Table 1). However, $47 \%$ of those who had delayed insulin treatment reported a diagnosis of type 2 diabetes, and $30 \%$ received co-treatment with oral glucose-lowering therapy; this is in marked contrast with the $7 \%$ who received oral glucose- 
Table 1 Comparison of the characteristics of study participants by diabetes classification and age of diagnosis

\begin{tabular}{|c|c|c|c|c|c|}
\hline Variable & $\begin{array}{l}\text { T1D diagnosed }>30 \\
\text { (C-peptide }<200 \\
\text { pmol/l, time to } \\
\text { insulin }<3 \text { years) }\end{array}$ & $\begin{array}{l}\text { T2D diagnosed }>30 \\
\text { (C-peptide } \\
\geq 600 \mathrm{pmol} / \mathrm{l})\end{array}$ & $\begin{array}{l}\text { T1D diagnosed } \leq 30 \\
\text { (C-peptide }<200 \\
\text { pmol/l, time to } \\
\text { insulin }<3 \text { years) }\end{array}$ & $\begin{array}{l}\text { T1D diagnosed } \\
>30 \text { vs T2D ( } p \text { value })\end{array}$ & $\begin{array}{l}\text { T1D diagnosed }>30 \\
\text { vs T1D diagnosed } \\
\leq 30 \text { ( } p \text { value })\end{array}$ \\
\hline Participant $(n)$ & 123 & 306 & 220 & - & - \\
\hline Current age (years) & $62(51-67)$ & $67(62-73)$ & $45(33-58)$ & $<0.001$ & $<0.001$ \\
\hline Age at diagnosis (years) & $44(36-54)$ & $54(46-60)$ & $16(11-22)$ & $<0.001$ & $<0.001$ \\
\hline Duration of diabetes at recruitment (years) & $13(6-22)$ & $13(10-19)$ & $26(14-40)$ & $>0.1$ & $<0.001$ \\
\hline BMI $\left(\mathrm{kg} / \mathrm{m}^{2}\right)$ & $25.9(23.0-29.4)$ & $31.6(28.1-36.2)$ & $26.0(23.3-28.7)$ & $<0.001$ & $>0.1$ \\
\hline Male sex $(\%)$ & $55(46,64)$ & $64(58,69)$ & $50(43,57)$ & 0.1 & $>0.1$ \\
\hline White ethnicity (\%) & $99(96,100)$ & $96(93,98)$ & $95(91,97)$ & $>0.1$ & $>0.1$ \\
\hline T1DGRS & $0.268(0.242-0.284)$ & $0.229(0.204-0.249)$ & $0.279(0.261-0.296)$ & $<0.001$ & $<0.001$ \\
\hline $\begin{array}{l}\text { T1DGRS }>5 \text { th centile of WTCCC } \\
\text { reference [12] }(\%)\end{array}$ & $82(74,88)$ & $45(40,51)$ & $96(92,98)$ & $<0.001$ & 0.001 \\
\hline C-peptide (pmol/l) & $7(3-65)$ & $1235(836-1770)$ & $4(3-13)$ & $<0.001$ & $<0.001$ \\
\hline Islet autoantibody positive (\%) & $78(67,87)$ & $6(3,11)$ & $62(53,69)$ & $<0.001$ & 0.02 \\
\hline $\begin{array}{l}\text { Concurrent use of oral } \\
\text { glucose-lowering agent }(\%)\end{array}$ & $15(10,23)$ & $80(75,84)$ & $5(2,8)$ & $<0.001$ & 0.001 \\
\hline Insulin at diagnosis $(\%)$ & $62(53,70)$ & $10(7,14)$ & $96(92,98)$ & $<0.001$ & $<0.001$ \\
\hline Time to insulin from diagnosis (months) & $0(0-3)$ & $60(24-120)$ & $0(0-0)$ & $<0.001$ & $<0.001$ \\
\hline $\begin{array}{l}\text { Basal bolus insulin regimen/insulin } \\
\text { pump (\%) }\end{array}$ & $85(77,92)$ & $16(11,21)$ & $94(90,97)$ & $<0.001$ & 0.01 \\
\hline Insulin dose (U/kg) & $0.62(0.46-0.83)$ & $0.50(0.30-0.86)$ & $0.59(0.44-0.82)$ & 0.02 & $>0.1$ \\
\hline $\mathrm{HbA}_{1 \mathrm{c}}(\mathrm{mmol} / \mathrm{mol})$ & $69(61-82)$ & $62(56-74)$ & $66(58-74)$ & $<0.001$ & 0.08 \\
\hline $\mathrm{HbA}_{1 \mathrm{c}}(\%)$ & $8.5(7.7-9.7)$ & $7.8(7.3-8.9)$ & $8.2(7.5-8.9)$ & $<0.001$ & 0.08 \\
\hline Self-reported T1D (\%) & $79(70,86)$ & $5(3,8)$ & $100(98,100)$ & $<0.001$ & $<0.001$ \\
\hline Self-reported T2D (\%) & $20(13,28)$ & $93(90,96)$ & $0(0,2)$ & $<0.001$ & $<0.001$ \\
\hline
\end{tabular}

Data are presented as $\%(95 \% \mathrm{CI})$ or median (IQR)

${ }^{a}$ Fifth centile of a young type 1 diabetes population corresponding to the 50th centile of a type 2 diabetes population [8]

IQR, interquartile range; T1D, type 1 diabetes; T2D type 2 diabetes; WTCCC, Wellcome Trust Case Control Consortium

lowering therapy in the group who received insulin from diagnosis $(p<0.01)$.

Early progression to insulin is a strong predictor of future severe insulin deficiency Eighty-five per cent (104/123) of all participants meeting criteria for type 1 diabetes (C-peptide $<200 \mathrm{pmol} / 1$ and insulin therapy within 3 years) in the cohort diagnosed $>30$ were treated with insulin within 1 year of diagnosis (78\% [104/133] for those defined by low Cpeptide only) vs $18 \%$ (55/306) of those meeting criteria for type 2 diabetes (Fig. 1). Thirty-two per cent $(37 / 115)$ of those with an intermediate C-peptide $(\geq 200-<600 \mathrm{pmol} / \mathrm{l})$ required insulin within 1 year of diagnosis. Of all those progressing to insulin within 3 years of diagnosis, $47 \%$ $(123 / 264)$ met the study criteria for type 1 diabetes and $18 \%(48 / 264)$ had intermediate C-peptide $(\geq 200-$ $<600 \mathrm{pmol} / \mathrm{l})$. Severe insulin deficiency was rare in those progressing to insulin after 3 years, occurring in only 10 of 231 participants (4\%; Fig. 1).

Thirty per cent of those diagnosed $>30$ years of age and administered insulin at diagnosis had type 2 diabetes according to C-peptide criteria Despite clear clinical, biochemical and genetic characteristics of type 2 diabetes (ESM Table 2), $30 \%$ of those meeting study criteria for type 2 diabetes were commenced on insulin at diagnosis. In the group meeting study criteria for type 2 diabetes and treated with insulin at diagnosis, $25 \%$ reported a diagnosis of type 1 diabetes, and only $59 \%$ received concurrent oral glucose-lowering therapy at a median diabetes duration of 10 years (ESM Table 2).

\section{Discussion}

To our knowledge, this is the first population analysis evaluating the prevalence of type 1 diabetes defined by low endogenous insulin secretion. Our study shows that late-onset type 1 diabetes with severe endogenous insulin deficiency is relatively common ( $21 \%$ of insulin-treated patients) and has very similar characteristics to young-onset type 1 diabetes, but is frequently initially treated as type 2 diabetes. Forty-seven per cent of participants progressing to insulin therapy within 3 years had type 1 diabetes and severe endogenous insulin deficiency, but this was often unrecognised.

Our finding that the clinical features of those with lateonset type 1 diabetes are similar to those with young-onset type 1 diabetes is consistent with recent research using a novel genetic stratification methodology [3]. This showed that $89 \%$ of those with genetically defined type 1 diabetes occurring after age 30 required insulin within a year, strikingly similar 


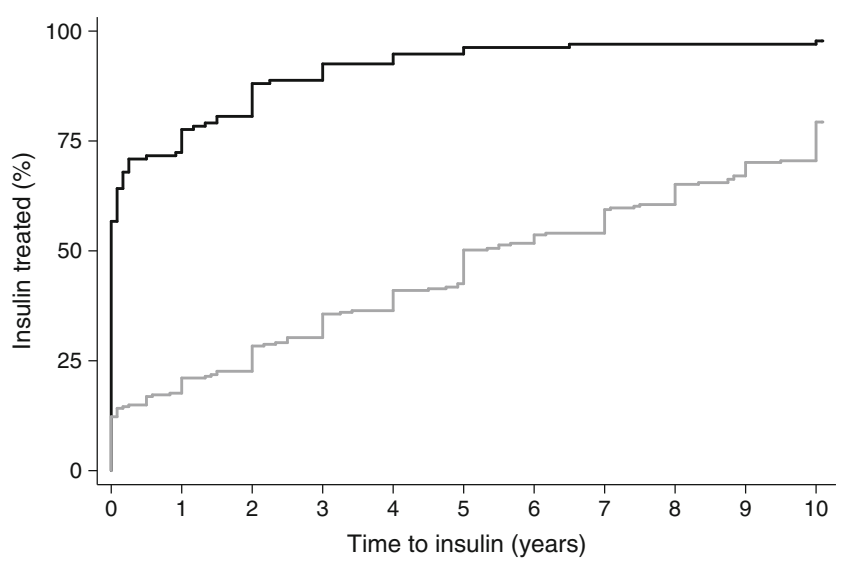

Fig. 1 Comparison of time to insulin therapy up to 10 years post diabetes diagnosis in participants with severe endogenous insulin deficiency (Cpeptide $<200 \mathrm{pmol} / \mathrm{l}$; black line) and participants with retained endogenous insulin secretion (C-peptide $\geq 600 \mathrm{pmol} / \mathrm{l}$; grey line)

to the $85 \%$ we report using a C-peptide-based definition. This is in contrast to diabetes defined solely by islet autoantibody status, where the phenotype appears intermediate between classical type 1 diabetes and type 2 diabetes in this age group [4].

A limitation of this study is the cross-sectional design within a relatively homogenous, geographically restricted population. Time to insulin and age of diagnosis were self-reported, and participants were assessed a median 13 years after diagnosis, meaning islet autoantibody positivity will be lower than at diagnosis [9]. Negative autoantibody tests in this context, therefore, do not exclude autoimmune diabetes, and we consider it likely that the aetiology of antibody-negative participants with low C-peptide will be autoimmune, as suggested by the high T1DGRS of these participants (median 0.262, data not shown). Islet autoantibodies were measured after a longer duration of diabetes in the young-onset cohort compared with those diagnosed later in life, potentially explaining the higher rate of islet autoantibody positivity observed in the late-onset cohort. A further limitation of this study is the lack of availability of a concurrent glucose level when C-peptide was measured; it is recognised that hypoglycaemia can result in a reduced C-peptide result [10].

These results have clear implications for clinical practice. They show that type 1 diabetes leading to endogenous insulin deficiency is common in later life but is difficult to identify. Consistent with this, many participants with type 1 diabetes in our cohort were diagnosed and treated as having type 2 diabetes. Without a diagnosis of type 1 diabetes, a patient will not receive appropriate education and will not be eligible for interventions that are often restricted to those with type 1 diabetes, such as carbohydrate counting, continuous glucose monitoring and insulin-pump therapy. They will be at risk of ketoacidosis if insulin is withdrawn. Our results suggest that if patients are treated as having type 2 diabetes but progress to insulin within 3 years of diagnosis, clinicians should reassess the underlying diagnosis and strongly consider biomarker testing $[5,8,11]$.

\section{Conclusion}

Type 1 diabetes defined by severe insulin deficiency has similar clinical and biological characteristics to type 1 diabetes occurring at younger ages. However, in later life, patients with type 1 diabetes leading to endogenous insulin deficiency are frequently diagnosed and treated as having type 2 diabetes. Clinicians should be aware that patients progressing to insulin within 3 years of diabetes diagnosis have a high likelihood of having type 1 diabetes, regardless of initial diagnosis.

Acknowledgements The authors thank participants who took part in the study and the research teams who undertook cohort recruitment. Data included in this article has previously been presented as an abstract at the 54th EASD Annual Meeting in 2018.

Data availability The datasets analysed during the current study are available through application to the Peninsula Research Bank, which is managed by the NIHR Exeter Clinical Research Facility. Information on application or data are available on http://exeter.crf.nihr.ac.uk/content/ tissue-banks

Funding The DARE study was funded by the Wellcome Trust and supported by the Exeter NIHR Clinical Research Facility. BMS and ATH are supported by the NIHR Exeter Clinical Research Facility. TJM is a National Institute for Health Research Senior Clinical Senior Lecturer. ATH is a Wellcome Trust Senior Investigator and NIHR Senior Investigator. RAO is supported by a Diabetes UK Harry Keen Fellowship (16/0005529). AGJ is supported by an NIHR Clinician Scientist award (CS-2015-15-018). The views given in this article do not necessarily represent those of the National Institute for Health Research, the National Health Service, or the Department of Health.

Duality of interest The authors declare that there is no duality of interest associated with this manuscript.

Contribution statement NJT, ATH and AGJ conceived the idea and designed the study. NJT, ALL, TJM, ATH, MNW, RAO, AVH, BMS and AGJ researched the data. NJT analysed the data with assistance from BMS and AGJ. NJT drafted the manuscript with assistance from AGJ. All authors critically revised the manuscript and approved the final version. AGJ is the guarantor of this work and, as such, had full access to all the data in the study and takes responsibility for the integrity of the data and the accuracy of the data analysis.

Open Access This article is distributed under the terms of the Creative Commons Attribution 4.0 International License (http:// creativecommons.org/licenses/by/4.0/), which permits unrestricted use, distribution, and reproduction in any medium, provided you give appropriate credit to the original author(s) and the source, provide a link to the Creative Commons license, and indicate if changes were made. 


\section{References}

1. American Diabetes Association (2017) Classification and diagnosis of diabetes. Diabetes Care 40(Supplement 1):S11-S24. https://doi. org $10.2337 / \mathrm{dc} 17-\mathrm{S} 005$

2. Shields BM, Peters JL, Cooper C et al (2015) Can clinical features be used to differentiate type 1 from type 2 diabetes? A systematic review of the literature. BMJ Open 5(11):e009088. https://doi.org/ 10.1136/bmjopen-2015-009088

3. Thomas NJ, Jones SE, Weedon MN, Shields BM, Oram RA, Hattersley AT (2018) Frequency and phenotype of type 1 diabetes in the first six decades of life: a cross-sectional, genetically stratified survival analysis from UK Biobank. Lancet Diabetes Endocrinol 6(2):122-129. https://doi.org/10.1016/S2213-8587(17)30362-5

4. Pozzilli P, Di Mario U (2001) Autoimmune diabetes not requiring insulin at diagnosis (latent autoimmune diabetes of the adult): definition, characterization, and potential prevention. Diabetes Care 24(8):1460-1467. https://doi.org/10.2337/diacare.24.8.1460

5. Jones AG, Hattersley AT (2013) The clinical utility of C-peptide measurement in the care of patients with diabetes. Diabet Med 30(7):803-817. https://doi.org/10.1111/dme.12159

6. Hope SV, Knight BA, Shields BM et al (2018) Random non-fasting C-peptide testing can identify patients with insulin-treated type 2 diabetes at high risk of hypoglycaemia. Diabetologia 61(1):66-74. https://doi.org/10.1007/s00125-017-4449-2
7. Grubb AL, McDonald TJ, Rutters F et al (2019) A type 1 diabetes genetic risk score can identify patients with GAD65 autoantibodypositive type 2 diabetes who rapidly progress to insulin therapy. Diabetes Care 42(2):208-214. https://doi.org/10.2337/dc18-0431

8. Oram RA, Patel K, Hill A et al (2016) A type 1 diabetes genetic risk score can aid discrimination between type 1 and type 2 diabetes in young adults. Diabetes Care 39(3):337-344. https://doi.org/10. 2337/dc15-1111

9. Williams GM, Long AE, Wilson IV et al (2016) Beta cell function and ongoing autoimmunity in long-standing, childhood onset type 1 diabetes. Diabetologia 59(12):2722-2726. https://doi.org/10. 1007/s00125-016-4087-0

10. Hope SV, Knight BA, Shields BM, Hattersley AT, McDonald TJ, Jones AG (2016) Random non-fasting C-peptide: bringing robust assessment of endogenous insulin secretion to the clinic. Diabet Med 33(11):1554-1558. https://doi.org/10.1111/dme.13142

11. Bingley PJ (2010) Clinical applications of diabetes antibody testing. J Clin Endocrinol Metab 95(1):25-33. https://doi.org/10.1210/jc. 2009-1365

12. Oram RA, Patel K, Hill A et al (2015) A type 1 diabetes genetic risk score can aid discrimination between type 1 and type 2 diabetes in young adults. Diabetes Care 39:337-344

Publisher's note Springer Nature remains neutral with regard to jurisdictional claims in published maps and institutional affiliations. 